\title{
A nonlinear Cournot duopoly with advertising
}

\author{
Luca Gori*• Mauro Sodini ${ }^{\dagger} \bullet$ Luciano Fanti $^{\ddagger}$
}

December 23, 2014

\begin{abstract}
This paper aims at studying local and global dynamics in a nonlinear duopoly with quantitysetting firms and non-cooperative advertising investments that affect the degree of (horizontally) differentiated products. It concentrates on persuasive advertising in a model where each firm has limited information and uses a behavioural rule to set the quantity for the subsequent period. By using some mathematical techniques and numerical simulations, our results show the existence of weak (à la Milnor) attractors, multistability and chaotic dynamics. In the long term, firms may continuously shift from states in which they invest in advertising to states in which advertising investment is absent.
\end{abstract}

Keywords Advertising; Chaos; Cournot duopoly; Local and global bifurcations

JEL Classification C62; D43; L13

\section{Introduction}

The aim of this paper is to build on a nonlinear duopoly (Bischi et al., 1998; Bischi et al., 2010) à la Cournot augmented with advertising investments - that affect the degree of (horizontally) differentiated products - to study long-term dynamics from both local and global perspectives. The importance of advertising as an instrument that allows firms to differentiate goods and get a competitive advantage on the market is well recognised (Bagwell, 2007). The industrial economics literature distinguishes between purely informative advertising (Grossman and Shapiro, 1984; Stahl, 1994; Hamilton, 2009) and persuasive advertising (Dixit and Norman, 1978). The former kind of investment provides information about price and product characteristics, but it does not influence the consumers' willingness to pay for the advertised good. As a consequence, it tends to favour competition, for instance by reducing search costs. The latter one consists in expenditures on interventions to persuade consumers that the own product is not perfectly substitutable with the rivals' one (Dixit and Norman, 1978). As a result, persuasive advertising is clearly anticompetitive because it tends to realise a spurious product differentiation, which in turn increases firms' market power. Product differentiation then represents a strategic variable especially in duopoly markets in

\footnotetext{
${ }^{*}$ L. Gori (corresponding author), Department of Law, University of Genoa, Via Balbi, 30/19, I-16126 Genoa (GE), Italy, e-mail: luca.gori@unige.it or dr.luca.gori@gmail.com, tel.: +390102099503, fax: +39010 2095536.

${ }^{\dagger}$ M. Sodini, Department of Economics and Management, University of Pisa, Via Cosimo Ridolfi, 10, I-56124 Pisa (PI), Italy, e-mail: mauro.sodini@unipi.it, tel.: +390502216 234, fax: +390502210603.

L. Fanti, Department of Economics and Management, University of Pisa, Via Cosimo Ridolfi, 10, I-56124 Pisa (PI), Italy, e-mail: luciano.fanti@unipi.it, tel.: +3905022 16 369, fax: +390502210603.
} 
both profit-maximising firms (Singh and Vives, 1984) and managerial firms (Kopel and Lambertini, 2013). Then studies that aim at clarifying the long-term behaviour of producers that operate in these kind of markets may be relevant also from a policy perspective.

This paper focuses on non-cooperative persuasive advertising in a nonlinear duopoly with quantity-setting firms. So far, the dynamic duopoly literature has dealt with similar issues in some works that concentrate on: 1) the relationship between advertising and goodwill (Luhta and Virtanen, 1996; Ahmed et al., 1999), or 2) the effect of brand competition on global dynamics in models where players have limited information (Bischi et al., 2000; Bischi and Kopel, 2003). However, a model that directly captures the long-term effects of advertising on product differentiation with quantity-setting firms is still lacking. The aim of this work is to fill this gap by considering players (firms) with limited information about rival's decision variables. Specifically, we assume that the degree of substitutability between products is affected by the level of advertising investment chosen by firms, and the larger such investment the more consumers perceive products as differentiated between each other. The assumption of limited information in a dynamic context follows several works in the related literature (Bischi et al., 1998; Bischi and Naimzada, 2000) and aims at capturing possible erratic behaviours of economic agents also when there are no exogenous stochastic shocks. Specifically, the assumption of full rationality implies that all agents have perfect knowledge about every detail of the game in which they are involved, and every agent knows that all others have perfect knowledge as well. In order to capture in a better way the complexity of "real world" situations (limited ability to compute optimal solutions, costs related to the enforcement of optimal plans and so on), the literature on nonlinear duopolies has relaxed this hypothesis by considering repeated instead of one-shot games and players whose strategies follow trial-and-error methods (for which less information than full rationality is required) and behave adaptively.

By considering feasibility constraints and the opportunity for firms to invest or not to invest in advertising, our model is described by a two-dimensional piecewise differentiable nonlinear map that shows some dynamic results related to chaos synchronisation and multistability (initial conditions matter). We have investigated bifurcations specifically related to piecewise maps along the diagonal and we have analysed the role of constraints in defining the basins of attractions of the system. The existence of constraints implies that the model is piecewise differentiable, so that possible border collision bifurcations may arise. In particular, we have concentrated on the existence of this kind of bifurcations related to the map restricted on the diagonal, by leaving to future research a deeper analysis of the role of points of non differentiability on the evolution of attractors that do not lie on the diagonal.

From an economic point of view, the model is able to generate long-term dynamics in which firms move from states where they decide to do not invest in advertising to states in which advertising investment is positive.

The rest of the paper proceeds as follows. Section 2 sets up the model. Section 3 takes a dynamic view of the problem and studies local stability properties. Section 4 concentrates on global analysis and provides some numerical experiments. Section 5 outlines the conclusions. The Appendix provides details of the static game and shows the existence of Nash equilibria in the cases of both positive advertising and no advertising.

\section{The model}

Consider an economy that consists of two types of agents: consumers and firms. There exists a competitive sector that produces the numeraire good $k \geq 0$ (whose price is normalised to 1 ), and 
a duopolistic sector with firm 1 and firm 2 that produce (horizontally) differentiated products of variety 1 and variety 2 , respectively. Let $p_{i} \geq 0$ and $q_{i} \geq 0$ be the price and quantity of product of firm $i(i=1,2)$, respectively.

Consumers are identical and their preferences towards goods $q_{1}, q_{2}$ and $k$ are captured by the separable utility function $V\left(q_{1}, q_{2}, k\right): R_{+}^{3} \rightarrow R_{+}$. They are specified by the following quasi-linear formulation: $V\left(q_{1}, q_{2}, k\right)=U\left(q_{1}, q_{2}\right)+k$, where $U\left(q_{1}, q_{2}\right): R_{+}^{2} \rightarrow R_{+}$is a twice differentiable function. The representative consumer maximises $V\left(q_{1}, q_{2}, k\right)$ subject to budget constraint $p_{1} q_{1}+$ $p_{2} q_{2}+k=M$, where $M>0$ is the exogenous nominal income of the consumer (assumed to be high enough to avoid the existence of corner solutions). Since $V\left(q_{1}, q_{2}, k\right)$ is a separable function and it is linear in $k$, there are no income effects on the duopolistic sector. The consumer's optimisation problem can then be written as: $\max _{\left\{q_{1}, q_{2}\right\}} U\left(q_{1}, q_{2}\right)-p_{1} q_{1}-p_{2} q_{2}+M$. By following Singh and Vives (1984), Häckner (2000) and Kopel and Lambertini (2013), we specify $U\left(q_{1}, q_{2}\right)$ as follows:

$$
U\left(q_{1}, q_{2}\right)=a\left(q_{1}+q_{2}\right)-\frac{1}{2}\left(q_{1}^{2}+q_{2}^{2}+2 d q_{1} q_{2}\right),
$$

where $a>0$ is the extent of market demand and $0<d \leq 1$ captures the degree of horizontal product differentiation. More in detail, if $d \rightarrow 0$ then products of variety 1 and variety 2 tend to be independent and each firm acts as a monopolist in its own market. For any $d<1$ products are perceived as imperfect substitutes by consumers and for $d=1$ they are perceived as perfect substitutes. By using (1) the consumer's maximisation programme gives the following inverse demands of goods 1 and 2, respectively:

$$
p_{1}=\max \left\{0, a-q_{1}-d q_{2}\right\} \quad \text { and } \quad p_{2}=\max \left\{0, a-q_{2}-d q_{1}\right\} .
$$

In order to guarantee that prices $p_{1}$ and $p_{2}$ are non-negative for any $d \in(0,1]$, quantities $q_{1}$ and $q_{2}$ must belong to set $A:=\left\{q_{1}, q_{2}: q_{1}+q_{2} \leq a\right\}$.

Firm 1 and firm 2 may differentiate their own product through advertising investment $z_{1}$ and $z_{2}$, respectively. Similar to Colangelo (1992), we assume the following specification of the degree of product differentiation $d$ :

$$
d=d\left(z_{1}, z_{2}\right):=\frac{1}{2\left(1+z_{1}\right)}+\frac{1}{2\left(1+z_{2}\right)},
$$

where $d \leq 1$ for any $z_{1}, z_{2} \geq 0$. The relationship between the degree of product substitutability and firm specific advertising investment described by (3) is made up to capture the effects of persuasive advertising on competition. In particular, the higher advertising investment, the lower consumers' ability to recognise product characteristics and the higher firm's market power.

Firms operate with the same marginal cost $w>0$, so that firm $i$ 's cost function is $c_{i}=w q_{i}+g z_{i}$ $(i=1,2)$, where $g>0$ is the cost per unit of advertising. Therefore, there exist constant marginal returns to labour and the production function is $q_{i}=L_{i}$, where $L_{i}$ is the labour force employed. For firm $i$, therefore, profits $\left(\Pi_{i}\right)$ are given by:

$$
\Pi_{i}=\left(p_{i}-w\right) q_{i}-g z_{i} .
$$

We note that (4) includes - through the expression of prices $p_{i}$ given by $(2)$ - the decision variables of the rival (firm $j$ ). Therefore, expectations of firm $i$ on the behaviour of the rival enter the profit maximisation programme. By considering the maximisation of $\Pi_{i}$ with respect to $z_{i}$ and making use of (2) and (3), we get:

$$
\max _{\left\{z_{i}\right\}} \Pi_{i}=\max _{\left\{z_{i}\right\}}\left\{\left(\max \left[0, a-q_{i}-d\left(z_{1}, z_{2}\right) q_{j}\right]-w\right) q_{i}-g z_{i}\right\},
$$


where $\Pi_{i}$ is a concave with respect to $z_{i}$. From the maximisation problem (5) we find the following expression for the optimal value of $z$ :

$$
z_{i}^{o p t}:=\max \left\{0, \sqrt{\frac{q_{i} q_{j}^{e}}{2 g}}-1\right\} .
$$

By substituting out $z_{i}^{\text {opt }}$ in $\Pi_{i}$ and assuming some restrictions on parameters in order to guarantee the concavity of the maximisation problem with respect to $q_{i}$, we get the optimal allocation $\left(q_{i}, z_{i}\right)$ for firm $i$ (see Colangelo, 1992), through which it is possible to define the Nash equilibrium of the model.

In the static game developed in this section and the Appendix, we have assumed that each firm knows the market demand and then proceeds with the calculation of the reaction function and the optimum in a strategic context. In the next section we will study the dynamics of a Cournot model with advertising by assuming discrete time and an adjustment mechanism of quantities based on a behavioural rule introduced when there is no perfect knowledge of the market (Bischi et al., 1998). To this purpose, we relax the assumption of knowledge of the market demand and use an adjustment mechanism based on marginal profits (Bischi and Naimzada, 2000).

\section{Dynamics}

At time $t=0$ both firms enter the market and choose the couple $\left(q_{i}, z_{i}\right)$. At this stage, they do not have information about the behaviour of the rival. We now introduce the following assumption:

Assumption A.1. $q_{i}(0)+q_{j}(0)<a$.

Assumption A.1 guarantees that prices are strictly positive at $t=0$. From now on, we avoid to report the time subscript when this does not cause ambiguity. Consider now a dynamic setting where time is discrete and indexed by $t=0,1,2, \ldots$ At every $t \geq 1$ both firms decide the quantity that should be produced for time $t+1$ by assuming that they have information about advertising investments. With regard to the rule used by firm $i$ at time $t \geq 1$ to determine $q_{i}$ in the subsequent period, we assume - by following Bischi et al. (1998) and Fanti et al. (2012, 2013) - that it has limited information (no knowledge on rival's decision variables) and chooses production for time $t+1$ through an adjustment mechanism based on its own marginal profit $\left(\partial \Pi_{i} / \partial q_{i}\right)$ at time $t$. In particular, once the choices of the rival about couple $\left(q_{j}, z_{j}\right)$ have been observed, firm $i$ uses the optimal rule (6) to compute its own profits and marginal profits by considering both rival's advertising investment and rival's production as given. We note that expression (6) is symmetric for both firms and $z_{i}$ is independent of $z_{j}$ for any $t \geq 1$. Then, either both firms will decide to make the strictly positive advertising investment $\sqrt{\frac{q_{1} q_{2}}{2 g}}-1$ or they do not invest in advertising.

From a mathematical point of view this implies that firm $i$ 's profits can be written as follows:

$$
\tilde{\Pi}_{i}=\Pi_{i}\left(q_{i}, q_{j}, z_{i}^{o p t}\left(q_{i}, q_{j}\right), z_{j}\right)=\left\{\begin{array}{c}
\Pi_{i}\left(q_{i}, q_{j}, \sqrt{\frac{q_{i} q_{j}}{2 g}}-1, z_{j}\right), \quad \text { if } \quad q_{i} \geq \frac{2 g}{q_{j}} \\
\Pi_{i}\left(q_{i}, q_{j}, 0,0\right), \quad \text { if } \quad q_{i}<\frac{2 g}{q_{j}}
\end{array} .\right.
$$


From straightforward calculations it is possible to verify that $\tilde{\Pi}_{i}$ is a continuous and differentiable function with respect to $q_{i}$ for any $q_{i}, q_{j} \geq 0$. Therefore, the marginal profit is:

$$
\frac{\partial \tilde{\Pi}_{i}}{\partial q_{i}}=\left\{\begin{array}{ccc}
M P_{i}^{A D V}:=a-w-2 q_{i}-\sqrt{\frac{2 g q_{j}}{q_{i}}}, & \text { if } \quad q_{i} \geq \frac{2 g}{q_{j}} \\
M P_{i}^{N O A D V}:=a-w-2 q_{i}-q_{j}, & \text { if } \quad q_{i}<\frac{2 g}{q_{j}}
\end{array},\right.
$$

where we have substituted (6) in rival's advertising expenditure $z_{j}$ after having computed the derivative, and $M P_{i}^{A D V}$ and $M P_{i}^{N O A D V}$ represent marginal profits under positive advertising investments and no advertising investments, respectively. Given firm $i$ 's own optimal advertising expenditure and given both the advertising expenditure and production of firm $j$, firm $i$ chooses the quantity that should be produced for time $t+1$ according to the rule introduced by Bischi and Naimzada (2000) when players have limited information. Therefore, the behaviour of production of firm $i$ from time $t$ to time $t+1$ is described by the following equation:

$$
q_{i}^{\prime}=q_{i}+\alpha q_{i} \frac{\partial \tilde{\Pi}_{i}}{\partial q_{i}}
$$

where $/$ is the unit-time advancement operator, $\alpha>0$ and $\partial \tilde{\Pi}_{i} / \partial q_{i}$ is determined by (8) depending on whether $q_{i} \geq \frac{2 g}{q_{j}}$ or $q_{i}<\frac{2 g}{q_{j}}$.

According to Fanti et al. (2013), where non-negative constraints on quantity are introduced, we have the following map:

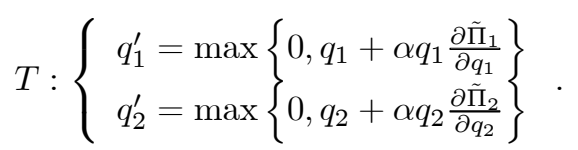

Let $M_{i}\left(q_{i}, q_{j}\right):=q_{i}+\alpha q_{i} M P_{i}^{A D V}$ and $N_{i}\left(q_{i}, q_{j}\right):=q_{i}+\alpha q_{i} M P_{i}^{N O A D V}$. Then, by following Radi et al. (2014) map $T$ is given by the following smooth maps defined in different subregions of the phase plane (see Figure 1, panel b):

$$
T_{\Omega_{1}}:\left\{\begin{array}{l}
q_{1}^{\prime}=M_{1}\left(q_{1}, q_{2}\right) \\
q_{2}^{\prime}=M_{2}\left(q_{1}, q_{2}\right)
\end{array} \quad \text { if } \quad\left(q_{1}, q_{2}\right) \in \Omega_{1}\right.
$$

where $\Omega_{1}:=\left\{\left(q_{1}, q_{2}\right) \in R_{+}^{2}: q_{1} q_{2} \geq 2 g, M_{1}\left(q_{1}, q_{2}\right) \geq 0, M_{2}\left(q_{1}, q_{2}\right) \geq 0\right\}$.

$$
T_{\Omega_{2}}:\left\{\begin{array}{l}
q_{1}^{\prime}=N_{1}\left(q_{1}, q_{2}\right) \\
q_{2}^{\prime}=N_{2}\left(q_{1}, q_{2}\right)
\end{array} \quad \text { if } \quad\left(q_{1}, q_{2}\right) \in \Omega_{2}\right.
$$

where $\Omega_{2}:=\left\{\left(q_{1}, q_{2}\right) \in R_{+}^{2}: q_{1} q_{2}<2 g, N_{1}\left(q_{1}, q_{2}\right) \geq 0, N_{2}\left(q_{1}, q_{2}\right) \geq 0\right\}$.

$$
T_{\Omega_{3}}:\left\{\begin{array}{c}
q_{1}^{\prime}=M_{1}\left(q_{1}, q_{2}\right) \\
q_{2}^{\prime}=0
\end{array} \quad \text { if } \quad\left(q_{1}, q_{2}\right) \in \Omega_{3}\right.
$$

where $\Omega_{3}:=\left\{\left(q_{1}, q_{2}\right) \in R_{+}^{2}: q_{1} q_{2} \geq 2 g, M_{1}\left(q_{1}, q_{2}\right) \geq 0, M_{2}\left(q_{1}, q_{2}\right)<0\right\}$.

$$
T_{\Omega_{4}}:\left\{\begin{array}{c}
q_{1}^{\prime}=0 \\
q_{2}^{\prime}=M_{2}\left(q_{1}, q_{2}\right)
\end{array} \quad \text { if } \quad\left(q_{1}, q_{2}\right) \in \Omega_{4}\right.
$$


where $\Omega_{4}:=\left\{\left(q_{1}, q_{2}\right) \in R_{+}^{2}: q_{1} q_{2} \geq 2 g, M_{1}\left(q_{1}, q_{2}\right)<0, M_{2}\left(q_{1}, q_{2}\right) \geq 0\right\}$.

$$
T_{\Omega_{5}}:\left\{\begin{array}{c}
q_{1}^{\prime}=N_{1}\left(q_{1}, q_{2}\right) \\
q_{2}^{\prime}=0
\end{array} \quad \text { if } \quad\left(q_{1}, q_{2}\right) \in \Omega_{5}\right.
$$

where $\Omega_{5}:=\left\{\left(q_{1}, q_{2}\right) \in R_{+}^{2}: q_{1} q_{2}<2 g, N_{1}\left(q_{1}, q_{2}\right) \geq 0, N_{2}\left(q_{1}, q_{2}\right)<0\right\}$.

$$
T_{\Omega_{6}}:\left\{\begin{array}{c}
q_{1}^{\prime}=0 \\
q_{2}^{\prime}=N_{2}\left(q_{1}, q_{2}\right)
\end{array} \quad \text { if } \quad\left(q_{1}, q_{2}\right) \in \Omega_{6},\right.
$$

where $\Omega_{6}:=\left\{\left(q_{1}, q_{2}\right) \in R_{+}^{2}: q_{1} q_{2}<2 g, N_{1}\left(q_{1}, q_{2}\right)<0, N_{2}\left(q_{1}, q_{2}\right) \geq 0\right\}$.

$$
T_{\Omega_{7}}:\left\{\begin{array}{l}
q_{1}^{\prime}=0 \\
q_{2}^{\prime}=0
\end{array} \quad \text { if } \quad\left(q_{1}, q_{2}\right) \in \Omega_{7},\right.
$$

where

$$
\begin{aligned}
\Omega_{7}: & =\left\{\left(q_{1}, q_{2}\right) \in R_{+}^{2}: q_{1} q_{2} \geq 2 g, M_{1}\left(q_{1}, q_{2}\right)<0, M_{2}\left(q_{1}, q_{2}\right)<0\right\} \cup \\
& \cup\left\{\left(q_{1}, q_{2}\right) \in R_{+}^{2}: q_{1} q_{2}<2 g, N_{1}\left(q_{1}, q_{2}\right)<0, N_{2}\left(q_{1}, q_{2}\right)<0\right\} .
\end{aligned}
$$

We note that for initial conditions that belong to sets $\Omega_{3}$ and $\Omega_{5}$ (resp. $\Omega_{4}$ and $\Omega_{6}$ ), dynamics definitely lie on $q_{2}$ (resp. $q_{1}$ ) semi-axis, that results to be forward invariant under $T$. This implies that if a trajectory ends up on one of the two axis, the subsequent iterates will lie on the same axis as well. Initial conditions that start from $\Omega_{7}$ generate trajectories mapped in $(0,0)$ in one iteration. In addition, depending on the value of $g$, some of sets $\Omega_{i}(i=1, \ldots, 7)$ may be empty. Specifically, if $g>\frac{a^{2}}{8}$ we get $\Omega_{2}=\Omega_{5}=\Omega_{6}=\emptyset$ (see Figure 1, panel a). More complicated is the behaviour of trajectories that start from initial conditions that belong to $\Omega_{1} \cup \Omega_{2}$. In fact, they can converge towards attractors that belong to $\Omega_{1} \cup \Omega_{2}$ or they can end up on non-negative semiaxes. In order to clarify this last result it is crucial the analysis through critical curves that will be developed subsequently.
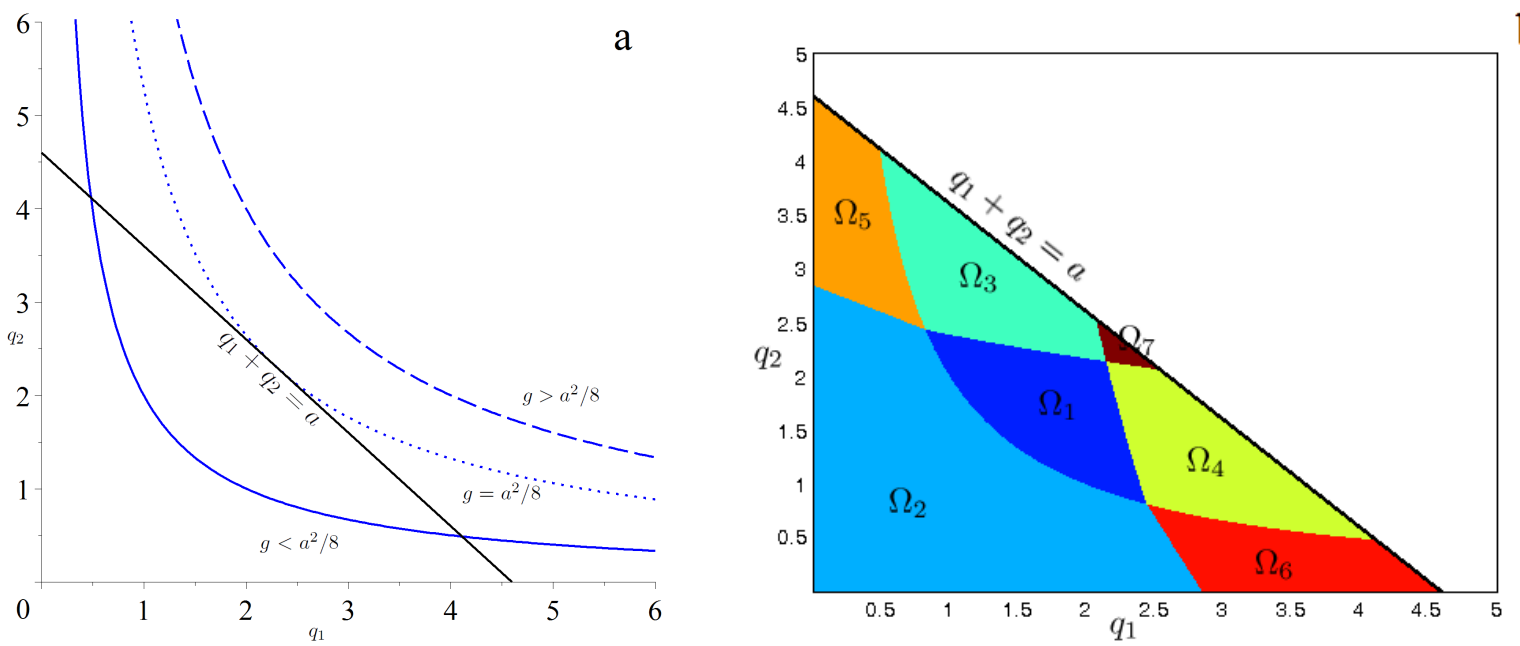
Figure 1. (a) Sets $\Omega_{1}, \Omega_{3}, \Omega_{4}$ and $\Omega_{7}$ lie at north-east with respect to curve $q_{1} q_{2}=2 g$. For sufficiently high values of $g$, that is $g>a^{2} / 8$ (the price of advertising is relatively high), curve $q_{1} q_{2}=2 g$ (dashed line) completely lies outside of triangle $A$ and then maps defined at least by one $M_{i}$ are not involved in the dynamics of the model. In this case the dynamics are described only by maps $T_{2}, T_{5}$ and $T_{6}$. The solid line describes case $g<a^{2} / 8$ (the price of advertising is relatively low) for which sets $\Omega_{i}$ are non-empty. The dotted line $\left(g=a^{2} / 8\right)$ represents the separating element between the two cases. (b) Case $g<a^{2} / 8$. Regions $\Omega_{i}(i=1, \ldots, 7)$ of the phase plane are evidenced by different colors. The dark-blue region is $\Omega_{1}$, the light-blue region is $\Omega_{2}$, the aqua green region is $\Omega_{3}$, the green region is $\Omega_{4}$, the orange region is $\Omega_{5}$, the red region is $\Omega_{6}$ and the blood-red region is $\Omega_{7}$.

With regard to the dynamic analysis at time $t=0$ (the time at which both firms enter the market), it is assumed that each firm does not know the quantity produced by the rival and then it cannot decide the optimal value of $z$ through equation (6). Therefore, at $t=0$ firm $i$ has to simultaneously choose both the quantity $q_{i}$ and advertising investment $z_{i}$. According to the partition of the phase plane and depending on expectations that firm $i$ has on the behaviour of firm $j$, at time $t=1$ the system will be in one of the region $\Omega_{i}$. Because of the dimension of the problem, we study the behaviour of the system (given the initial conditions) on the projection of the space $\left(q_{1}(0), q_{2}(0), z_{1}(0), z_{2}(0)\right)$ on plane $\left(q_{1}(0), q_{2}(0)\right)$, that is by fixing initial conditions on advertising investment $z_{i}$ and $z_{j}$ we characterise the initial conditions on quantities $q_{i}$ and $q_{j}$ such that the system will be in one of sets $\Omega_{i}$. Specifically, given the couple of initial conditions $\left(z_{1}(0), z_{2}(0)\right)$, then through the use of

$$
\left.\left\{\begin{array}{l}
q_{1}(1)=\left\{0, q_{1}(0)+\alpha q_{1}(0)\left[a-w-2 q_{1}(0)-\frac{1}{2}\left(\frac{1}{1+z_{1}(0)}+\frac{1}{1+z_{2}(0)}\right) q_{2}(0)\right.\right. \\
q_{2}(1)=\left\{0, q_{2}(0)+\alpha q_{2}(0)\left[a-w-2 q_{2}(0)-\frac{1}{2}\left(\frac{1}{1+z_{1}(0)}+\frac{1}{1+z_{2}(0)}\right) q_{1}(0)\right.\right.
\end{array}\right]\right\},
$$

it is possible to build on the subset of initial conditions $\left(q_{1}(0), q_{2}(0)\right)$ (eventually empty) that verifies Assumption A.1 and guarantees that iterate $\left(q_{1}(1), q_{2}(1)\right)$ belongs to $\Omega_{i}$, where the expression in (18) defines the iterate at time $t=0$ of the map. In addition, we note that $z_{1}(0)$ and $z_{2}(0)$ can be positive (both firms invest in advertising at time $t=0$ ), zero (both firms do not invest in advertising at time $t=0$ ) or, alternatively, $z_{1}(0)=0$ and $z_{2}(0)>0$ or viceversa. Figure 2 panel (a) (resp. panel (b)) shows in the $\left(q_{1}(0), q_{2}(0)\right)$ plane the region $\Omega_{i}$ in which the system will be located at time $t=1$ if each firm expects that the rival will not invest (resp. will invest) in advertising. In other words, in Figures 2.a and 2.b the preimages of order 1 through $T$ of regions $\Omega_{i}$ are depicted. 
$\mathrm{a}$
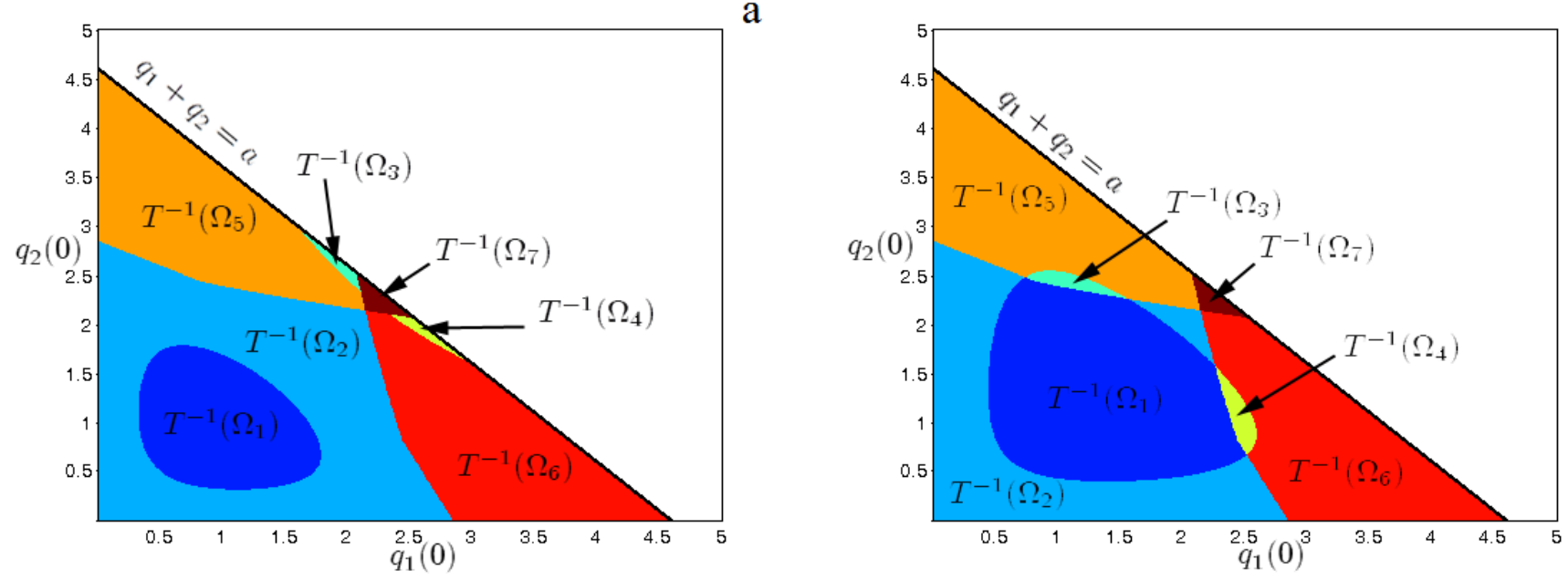

Figure 2. (a) At time $t=0$, each firm chooses quantity and advertising investment by expecting no advertising investments by the rival. (b) At time $t=0$, each firm chooses quantity and advertising investment by expecting positive advertising investments by the rival. Different colours evidence different behaviours of map $T$ at $t=0$. Colours in Figures 2.a and 2.b correspond to colours in Figure 1.b. Specifically, an initial condition $\left(q_{1}(0), q_{2}(0)\right)$ that starts from the dark-blue region in both figures is mapped into $\Omega_{1}$ at time $t=1$ (a similar behaviour holds for other colours).

Map $T$ has the non-interior fixed points

$$
E_{0}=(0,0), \quad E_{1}=\left(\frac{a-w}{2}, 0\right), \quad E_{2}=\left(0, \frac{a-w}{2}\right)
$$

that are fixed points also for maps $T_{\Omega_{1}}, T_{\Omega_{5}}$ and $T_{\Omega_{6}}$. We now state the following proposition.

Proposition 1 Let $a-w>0$. Then, map $T$ admits a unique interior fixed point $E^{*}$, where

$$
E^{*}=\left\{\begin{array}{c}
\left(\frac{a-w-\sqrt{2 g}}{2}, \frac{a-w-\sqrt{2 g}}{2}\right), \quad \text { if } \quad g \leq \frac{(a-w)^{2}}{18} \\
\left(\frac{a-w}{3}, \frac{a-w}{3}\right), \quad \text { if } \quad g>\frac{(a-w)^{2}}{18}
\end{array} .\right.
$$

Proof. First, we note that an interior fixed point of $T$ is a fixed point of map $T_{\Omega_{1}}$ or map $T_{\Omega_{2}}$. If the fixed point belongs to $\Omega_{2}$, its coordinates are solution in $\Omega_{2}$ of

$$
\left\{\begin{array}{l}
M P_{1}^{N O A D V}=0 \\
M P_{2}^{N O A D V}=0
\end{array}\right.
$$

From (21), we find $\left(\frac{a-w}{3}, \frac{a-w}{3}\right)$ which is feasible if $g>\frac{(a-w)^{2}}{18}$. If the fixed point belongs to $\Omega_{1}$, we have to solve

$$
\left\{\begin{array}{l}
M P_{1}^{A D V}=0 \\
M P_{2}^{A D V}=0
\end{array}\right.
$$


which is equivalent to

$$
\left\{\begin{array}{c}
M P_{1}^{A D V}=0 \\
\frac{q_{2}}{q_{1}}=\frac{a-w-2 q_{1}}{a-w-2 q_{2}}
\end{array} .\right.
$$

From (23) we have the following couple of solutions (and their symmetric counterparts)

$$
\left(\frac{a-w}{2}, 0\right), \quad\left(\frac{a-w}{4}-\frac{\sqrt{2}}{8} \sqrt{2(a-w)^{2}-16 g}, \frac{a-w}{4}+\frac{\sqrt{2}}{8} \sqrt{2(a-w)^{2}-16 g}\right),
$$

that do not belong to $\Omega_{1}$, and $\left(\frac{a-w-\sqrt{2 g}}{2}, \frac{a-w-\sqrt{2 g}}{2}\right)$ which is feasible if $g \leq \min \left\{\frac{(a-w)^{2}}{18}, \frac{(a-w)^{2}}{2}\right\}=$ $\frac{(a-w)^{2}}{18}$.

We now have the following remark.

Remark 2 Fixed point $E^{*}$ coincides with coordinates $\left(q_{1}^{*}, q_{2}^{*}\right)$ of the Nash equilibrium $\left(q_{1}^{*}, z_{1}^{*}, q_{2}^{*}, z_{2}^{*}\right)$ for the static game, where $z_{i}^{*}=z_{i}^{\text {opt }}\left(q_{i}^{*}\right)$. As a consequence, if $g \leq \frac{(a-w)^{2}}{18}$ (resp. $g>\frac{(a-w)^{2}}{18}$ ) firms invest (resp., do not invest) in advertising at stationary state $E^{*}$.

In order to study the local properties of the model around the stationary equilibria, we have to compute the Jacobian matrix. Specifically, the generic Jacobian matrix for map $T$ is the following:

$$
J\left(q_{1}, q_{2}\right)=\left(\begin{array}{cc}
-4 \alpha q_{1}+1+\alpha(a-w)-\alpha \sqrt{\frac{g q_{2}}{2 q_{1}}} & -\alpha \sqrt{\frac{g q_{1}}{2 q_{2}}} \\
-\alpha \sqrt{\frac{g q_{2}}{2 q_{1}}} & -4 \alpha q_{2}+1+\alpha(a-w)-\alpha \sqrt{\frac{g q_{1}}{2 q_{2}}}
\end{array}\right),
$$

if $\left(q_{1}, q_{2}\right) \in \operatorname{int}\left(\Omega_{1}\right)$, while we have that

$$
J\left(q_{1}, q_{2}\right)=\left(\begin{array}{cc}
-4 \alpha q_{1}+1+\alpha(a-w)-\alpha q_{2} & -\alpha q_{1} \\
-\alpha q_{2} & -4 \alpha q_{2}+1+\alpha(a-w)-\alpha q_{1}
\end{array}\right),
$$

if $\left(q_{1}, q_{2}\right) \in \operatorname{int}\left(\Omega_{2}\right)$.

Then, we can easily find that $E_{0}$ is unstable, and $E_{1}$ and $E_{2}$ are saddles. In order to characterise the dynamic properties of $E^{*}$, a useful starting point is the analysis of the behaviour of the map restricted on the diagonal. In particular, we will concentrate on how a change in parameter $g$ (that represents the price per unit of advertising investment) affects the dynamics of the model. Map $T$ is symmetric, i.e. it does not change if variables $q_{1}$ and $q_{2}$ are swapped, that is $T \circ S=S \circ T$ , where $S:\left(q_{1}, q_{2}\right) \rightarrow\left(q_{2}, q_{1}\right)$. This implies that the diagonal $\Delta=\left\{\left(q_{1}, q_{2}\right): q_{1}=q_{2}\right\}$ is an invariant manifold, i.e. by starting from $q_{1}(0)=q_{2}(0)$ the dynamics lie on $\Delta$ for every $t$. In this case, the behaviour of the dynamic system is characterised by the restriction of map $T$ on $\Delta$, and synchronised trajectories (i.e., $q_{1}(t)=q_{2}(t)$ for every $t$ ) are governed by $T_{\Delta}: \Delta \rightarrow \Delta$, where

$$
T_{\Delta}: q^{\prime}:=f(q)=\left\{\begin{array}{cll}
f_{1}(q):=q+\alpha q(a-w-2 q-\sqrt{2 g}) & \text { if } \quad q \geq \sqrt{2 g} \\
f_{2}(q):=q+\alpha q(a-w-3 q) & \text { if } & q<\sqrt{2 g}
\end{array},\right.
$$

is the map restricted on the diagonal (by ignoring the non-negativity constraints). 
We note that each piece in (27) resembles the logistic map. More specifically, the former (resp. latter) piece is conjugated with the logistic map $y^{\prime}=\mu y(1-y)$ by the linear transformation $q=\frac{1+\alpha(a-w-\sqrt{2 g})}{2 \alpha} y$ with $\mu=1+\alpha(a-w-\sqrt{2 g})\left(\right.$ resp. $q=\frac{1+\alpha(a-w)}{3 \alpha} y$ with $\left.\mu=1+\alpha(a-w)\right)$. In particular, if

$$
g>\frac{[1+\alpha(a-w)]^{2}}{18 \alpha^{2}}:=\tilde{g},
$$

and

$$
\alpha(a-w)<3,
$$

bounded dynamics are completely defined by the second equation in (27). We note that the dynamic properties of map (27) are different than the traditional results of the logistic map because of the non-differentiability of $T_{\Delta}$ at $q=\sqrt{2 g}$. Specifically, multiple attractors with non-connected basins can exist. In addition, the unitary cost of advertising $(g)$ enters the definition of both equations in (27) and the definition of the threshold of the map. Thus, a change in $g$ causes several changes that may be difficult to be classified. In contrast, if $g<\tilde{g}$ the map restricted on the diagonal is properly smooth piecewise since both branches of map $T_{\Delta}$ are involved. In this case, the map continues to be unimodal. In fact, if $f \prime_{2}(\sqrt{2 g})=1+\alpha(a-w-6 \sqrt{2 g})<0$ then $f \prime_{1}(q)<0$ for any $q>\sqrt{2 g}$. Let $q_{\max }:=\frac{1+\alpha(a-w)}{6 \alpha}$ be a threshold value of the quantity produced by both firms. Then, we can have three different cases: 1 ) if $g>\frac{1}{2}\left[\frac{1+\alpha(a-w)}{6 \alpha}\right]^{2}$ the maximum point belongs to $f_{2}$; 2) if $g<\frac{1}{2}\left[\frac{1+\alpha(a-w)}{6 \alpha}\right]^{2}$ the maximum point belongs to $\left.f_{1} ; 3\right)$ if $g=\frac{1}{2}\left[\frac{1+\alpha(a-w)}{6 \alpha}\right]^{2}$ the maximum point corresponds to the point in which the map is not differentiable.

We recall that the maximum point and the point at which the map is not differentiable play an important role in the definition of the absorbing areas. We now wonder whether by starting from an initial condition that does not lie on the diagonal, the dynamics converge towards it or, alternatively the dynamics do not synchronise, i.e. we can have a coordination failure. Then, by considering the restriction of map $T$ on a generic point on $\Delta$, we have the following Jacobian matrix:

$$
J(q, q)=\left(\begin{array}{cc}
l(q) & m(q) \\
m(q) & l(q)
\end{array}\right),
$$

which is differently defined depending on whether $\left(q_{1}, q_{2}\right) \in \operatorname{int}\left(\Omega_{1}\right)$ or $\left(q_{1}, q_{2}\right) \in \operatorname{int}\left(\Omega_{2}\right)$. In particular,

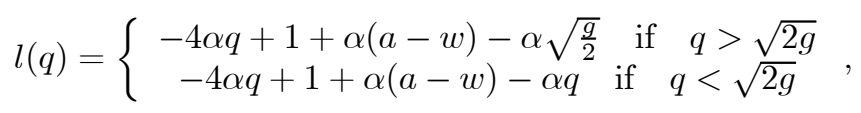

and

$$
m(q)=\left\{\begin{array}{c}
-\alpha \sqrt{\frac{g}{2}} \text { if } q>\sqrt{2 g} \\
-\alpha q \text { if } q<\sqrt{2 g}
\end{array} .\right.
$$

The eigenvalues associated to a generic point on $\Delta$ are the following:

$$
\lambda_{\|}=l(q)+m(q)=\left\{\begin{array}{c}
-4 \alpha q+1+\alpha(a-w)-2 \alpha \sqrt{\frac{g}{2}} \quad \text { if } \quad q>\sqrt{2 g} \\
-4 \alpha q+1+\alpha(a-w)-2 \alpha q \quad \text { if } \quad q<\sqrt{2 g},
\end{array},\right.
$$

with eigenvector $(1,1)$ and

$$
\lambda_{\perp}=l(q)-m(q)=\left\{\begin{array}{lll}
-4 \alpha q+1+\alpha(a-w) & \text { if } & q>\sqrt{2 g} \\
-4 \alpha q+1+\alpha(a-w) & \text { if } & q<\sqrt{2 g}
\end{array},\right.
$$


with eigenvector $(1,-1)$. The eigenvalue $\lambda_{\|}$is related to the invariant manifold $\Delta$ and coincides with the multiplier of the restriction of the map on $\Delta$. The eigenvector associated with the other eigenvalue is always orthogonal to $\Delta$ regardless of $q$.

Through the study of the eigenvalues it is possible to characterise the bifurcations that occur on the diagonal.

Proposition 3 The fixed point $E^{*}$ is locally asymptotically stable if: 1) $g<\frac{2}{9 \alpha^{2}}$ and $0<a-w<$ $\frac{2}{\alpha}+\sqrt{2 g}$; 2) $g>\frac{2}{9 \alpha^{2}}$ and $a-w \in V$, where $V=\left(0, \frac{2}{\alpha}\right) \cup\left(3 \sqrt{2 g}, \frac{2}{\alpha}+\sqrt{2 g}\right)$. The fixed point $E^{*}$ undergoes a supercritical flip bifurcation when: a) $g<\frac{2}{9 \alpha^{2}}$ and $a-w=\frac{2}{\alpha}+\sqrt{2 g}$; b) $g>\frac{2}{9 \alpha^{2}}$ and $a-w=\frac{2}{\alpha}$; c) $g>\frac{2}{9 \alpha_{2}^{2}}$ and $a-w=\frac{2}{\alpha}+\sqrt{2 g}$. The fixed point $E^{*}$ undergoes a border collision bifurcation when $g>\frac{2}{9 \alpha^{2}}$ and $a-w=3 \sqrt{2 g}$.

Proof. If $E^{*} \in \operatorname{int}\left(\Omega_{1}\right)$, that is $g<\frac{1}{18}(a-w)^{2}$, the Jacobian matrix is the following:

$$
J\left(E^{*}\right)=\left(\begin{array}{cc}
1-\alpha\left(a-w-\frac{3}{2} \sqrt{2 g}\right) & -\frac{1}{2} \alpha \sqrt{2 g} \\
-\frac{1}{2} \alpha \sqrt{2 g} & 1-\alpha\left(a-w-\frac{3}{2} \sqrt{2 g}\right)
\end{array}\right),
$$

In contrast, if $E^{*} \in \operatorname{int}\left(\Omega_{2}\right)$, that is $g>\frac{1}{18}(a-w)^{2}$, we get:

$$
J\left(E^{*}\right)=\left(\begin{array}{cc}
1-\frac{2}{3} \alpha(a-w) & -\frac{1}{3} \alpha(a-w) \\
-\frac{1}{3} \alpha(a-w) & 1-\frac{2}{3} \alpha(a-w)
\end{array}\right) .
$$

By combining the conditions for the existence of the fixed points and the stability conditions given by the eigenvalues of Jacobian matrices (35) and (36), we get the result.

Remark 4 We note that if $g>\frac{1}{2 \alpha^{2}}$ the second set in $V$ is empty.

The results of Proposition 3 can be generalised when an attracting cycle for $T_{\Delta}$ on $\Delta$ does exist. We now recall that for a $k$-cycle $\left\{\left(q_{1}, q_{1}\right), \ldots,\left(q_{m}, q_{m}\right)\right\}$ of $T$ embedded into the invariant line $\Delta$ and corresponding to cycle $\left\{q_{1}, \ldots, q_{m}\right\}$ of $f$, multipliers are given by

$$
\lambda_{\|}^{k}=\prod_{i=1}^{k}\left(l\left(q_{i}\right)+m\left(q_{i}\right)\right),
$$

with eigenvector $(1,1)$ and

$$
\lambda_{\perp}^{k}=\prod_{i=1}^{k}\left(l\left(q_{i}\right)-m\left(q_{i}\right)\right)
$$

with eigenvector $(1,-1)$. Stability is ensured by the condition $\left|\lambda_{\|}^{k}\right|<1$. We recall that an attractor $X$ of $f$ is an asymptotically stable attractor of $T$ if and only if all trajectories that belong to $X$ are transversely attracting. In particular, if $X$ is a chaotic attractor a stability condition can be given in terms of the transverse Lyapunov exponent 


$$
\Lambda_{\perp}=\lim _{n \rightarrow \infty} \frac{1}{n} \sum_{i=1}^{n} \ln \left|\lambda_{\perp}(q(i))\right|,
$$

where $q(0) \in A$ and $q(i)$ is the trajectory generated by $f$. The following classical definition of attractiveness can be established

Definition $5 X$ is an asymptotically stable attractor (or topological attractor) if it is Lyapunov stable, i.e. for every neighbourhood $U$ of $X$ there exists a neighbourhood $V$ of $X$ such that $T^{n}(V) \subset$ $U$ for every $n \geq 0$ and the basin of attraction $B(X)$ contains a neighbourhood of $X$.

According to the initial conditions, it is possible to define a spectrum of Lyapunov exponents

$$
\Lambda_{\perp}^{\min }<\ldots<\Lambda_{\perp}^{\text {nat }}<\ldots \Lambda_{\perp}^{\max },
$$

where $\Lambda_{\perp}^{\text {nat }}$ is the Lyapunov exponent evaluated on a generic trajectory taken in the chaotic attractor. If a set is a Lyapunov attractor then $\Lambda_{\perp}^{\max }<0$. If $\Lambda_{\perp}^{\max }>0$ and $\Lambda_{\perp}^{\text {nat }}<0$, the set is no longer Lyapunov stable but it attracts a large set (the basin of attraction has positive Lebesgue measure) of points in the two-dimensional phase space. In this case, $X$ is a Milnor attractor.

Definition 6 A closed invariant set $X$ is said to be a weak attractor in Milnor sense if its stable set $B(X)$ has positive Lebesgue measure.

Finally, if $\Lambda_{\perp}^{\text {nat }}>0$ then $X$ is a chaotic saddle and trajectories that start from initial conditions close to the diagonal can either be captured by an attractor that envelops the saddle or captured by other attractors.

In order to stress the interplay between the behaviours of the system on the diagonal and those that involve the whole phase plane, in what follows we will investigate both phenomena at the same time.

\section{Global analysis and numerical simulations}

This section is concerned with the study of some local and global phenomena related to map $T$. In particular, we will deepen the analysis of: 1) the possible (loss of) synchronisation in the dynamics of the model and coexistence of attractors, 2) the existence of bifurcations related to the nondifferentiability of the map, and 3) the evolution of the basins of attraction. In order to preserve the non-negativity of prices, in the following numerical experiments we will consider parameter sets for which $\Omega_{1} \cup \Omega_{2}$ are subset of $A$.

By using arguments similar to those in Bischi et al. (1998) and Fanti et al. (2012), the following proposition on the boundaries of basins of attraction can be stated. 
Proposition 7 Let $S$ be the union of attractors that lie on non-negative semiaxes. If $0<\alpha(1-w)<$ 3 then

$$
\partial B(S)=\left(\cup_{n=0}^{+\infty} T^{-n}\left(\omega_{1}\right)\right) \cup\left(\cup_{n=0}^{+\infty} T^{-n}\left(\omega_{2}\right)\right),
$$

where $\omega_{1}$ (resp. $\left.\omega_{2}\right)$ is the segment line whose coordinates are $(0,0)$ and $\left(\frac{1+\alpha(1-w)}{2 \alpha}, 0\right)($ resp. $(0,0)$ and $\left.\left(0, \frac{1+\alpha(1-w)}{2 \alpha}\right)\right)$.

Proof. See Bischi et al. (1998).

This finding allows us to analytically define the borders of region $\cup_{i=3}^{7} \Omega_{i}$. In particular, it is possible to identify the set of initial conditions such that trajectories definitely lie on the nonnegative semiaxes, that is the initial conditions whose trajectories imply that at least one of the two firms exits the market. We note that on the non-negative semiaxes only the definitions of $T_{\Omega_{2}}$, $T_{\Omega_{5}}$ and $T_{\Omega_{6}}$ hold. From an economic point of view, this implies that when one of the two firms exits the market the other one does not invest in advertising. In this case, the dynamics of variable $q_{-i}$ (that is, the quantity produced by the firm that remains on the market) is described by the equation

$$
q_{-i}^{\prime}=\max \left\{0, q_{-i}+\alpha q_{-i}\left(a-w-2 q_{-i}\right)\right\},
$$

which is conjugated to the logistic map (see Bischi et al., 1998 for details) and can have either dynamics convergent to $(a-w) / 2$ (the non null coordinate of fixed point $E_{-i}$ ) or cyclical or chaotic dynamics.

In what follows, we perform the global analysis of map $T$ through the use of some numerical experiments reported in Cases 1-3.

Case 1. The parameter set is the following: $\alpha=0.64, a=4.6, w=0.4$ and $g=0.8$. First, we note that $E^{*} \in \Omega_{1}$ is the unique attractor (with positive coordinates) for points that start on set $\operatorname{int}\left(\Omega_{1} \cup \Omega_{2}\right)$. With this parameter values, the Nash equilibrium implies positive advertising investments for both firms (see Figure 3.a that describes the dynamics on the diagonal). However, there also exist attractors $E_{0}, E_{1}$ and $E_{2}$ that respectively capture the dynamics that start from initial conditions that lie on the sets represented by the red, light-grey and yellow coloured regions in Figure 3.b. The bound of the basin has been obtained with techniques similar to those used by Bischi et al. (1998). In particular, in this case it can be shown that the lines (preimages) that mark the borders between $\Omega_{1} \cup \Omega_{2}$ and $\cup_{i=3}^{7} \Omega_{i}$ also define the basin of attraction of $E^{*}$. 


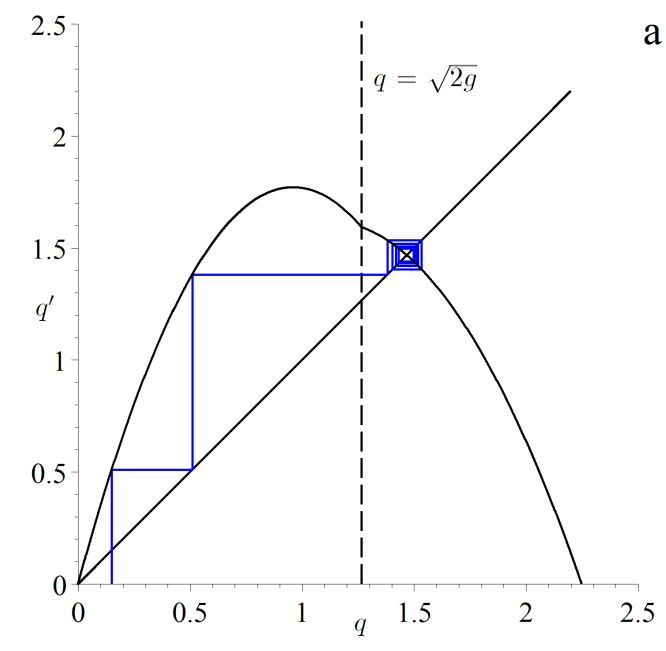

a

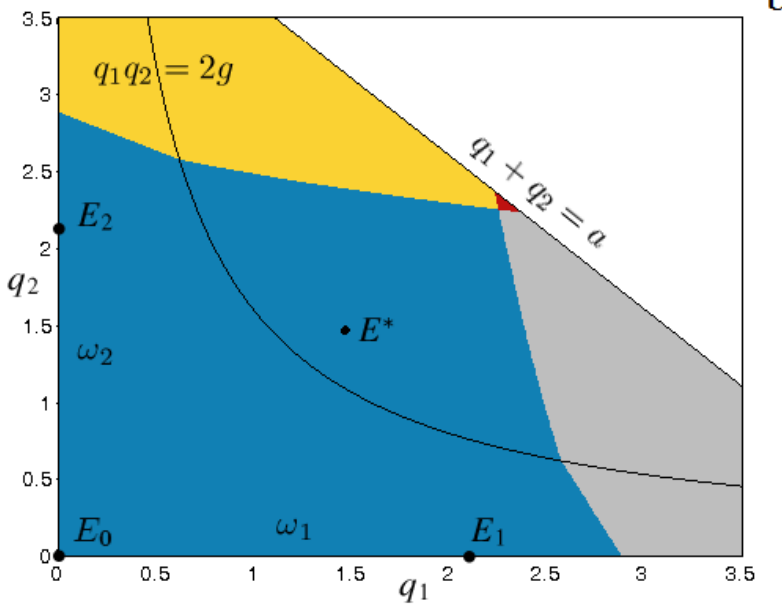

Figure 3. (a) Dynamics associated to map $T_{\Delta}$. (b) Basins of attraction of the fixed points of map $T$ (see the main text for details).

Case 2. Let the parameter set be $\alpha=0.67, a=4.6, w=0.4$ (this also holds for Case 3) and let $g$ vary. Figure 4.d shows the evolution of the transverse Lyapunov exponent for $g$. In particular, when $g=1.31$ we have a chaotic attractor for the map restricted on the diagonal that captures (in the Milnor sense) all trajectories that start from $\operatorname{int}\left(\Omega_{1} \cup \Omega_{2}\right)$ (see Figures 4.a and 4.b). This is pointed out in Figure 4.c that shows a long transient together with some burst away from the diagonal that eventually lead to synchronisation of trajectories towards it (on-off intermittency). For lower values of $g$ (1.305) the Lyapunov exponent is positive. Then, we have the loss of synchronisation (Figure 4.e) and the attractor does not lie on the diagonal anymore.

We note that for the different values of $g$ considered in Case 2, it is possible to obtain through the theory of critical curves 1) the absorbing area in which trajectories are bounded during several bursts away from the diagonal before the synchronisation $(g=1.31)$, or 2$)$ the absorbing area completely fill up by the chaotic attractor after the loss of synchronisation (see Figure 4.f, plotted for $g=1.305$, where the boundaries of the basin of attraction have been obtained through the use of Proposition 7).

It is important to stress that the synchronisation failure holds in spite of the hypothesis of homogeneous players (i.e., when firms use the same technology and behavioural rule). Furthermore, it is interesting to note that when $g$ changes the economy continuously alternates between long time periods in which both firms do not invest in advertising and long time periods in which they invest in advertising. 


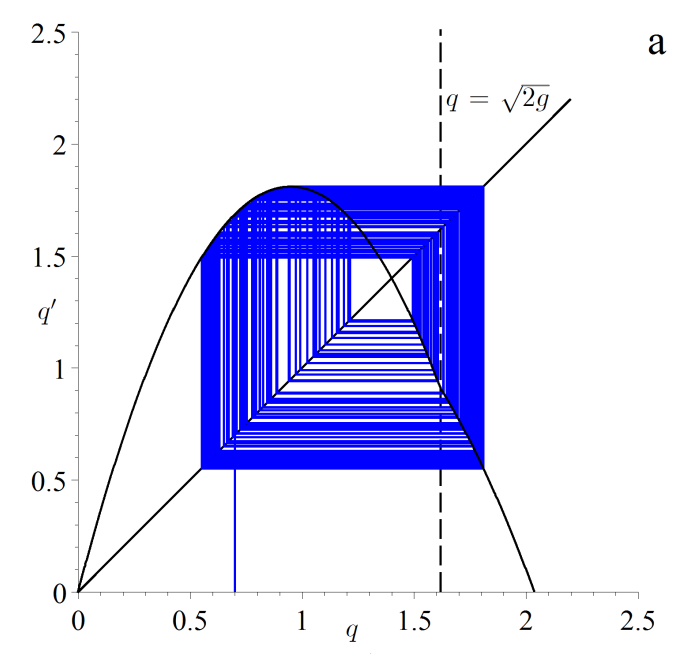

a


c

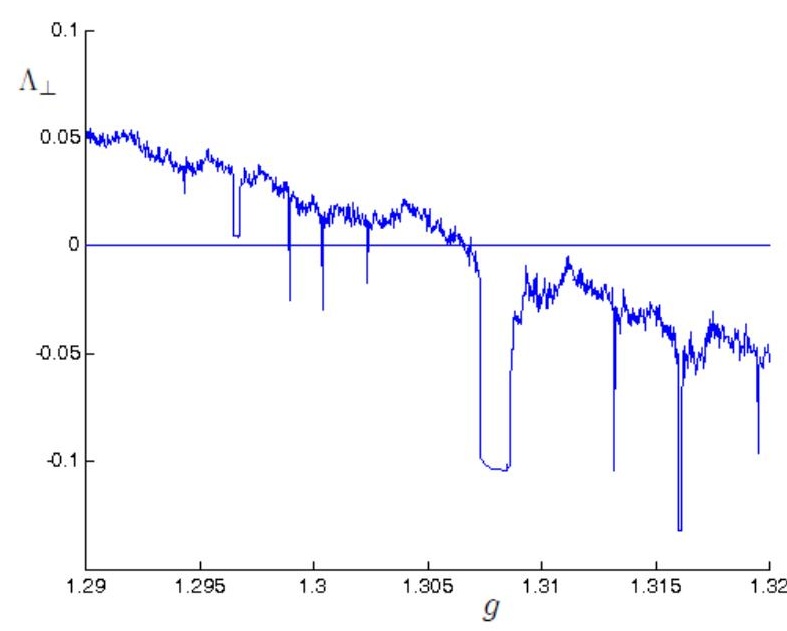




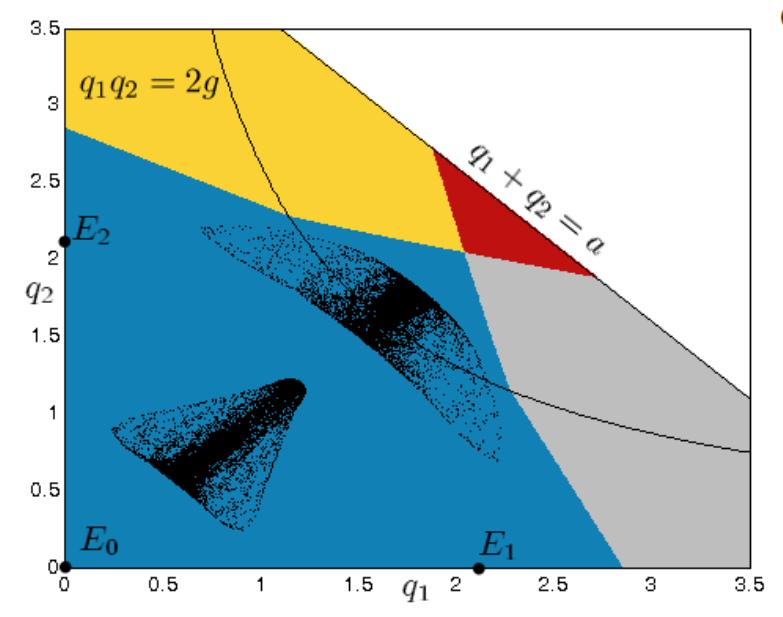

e

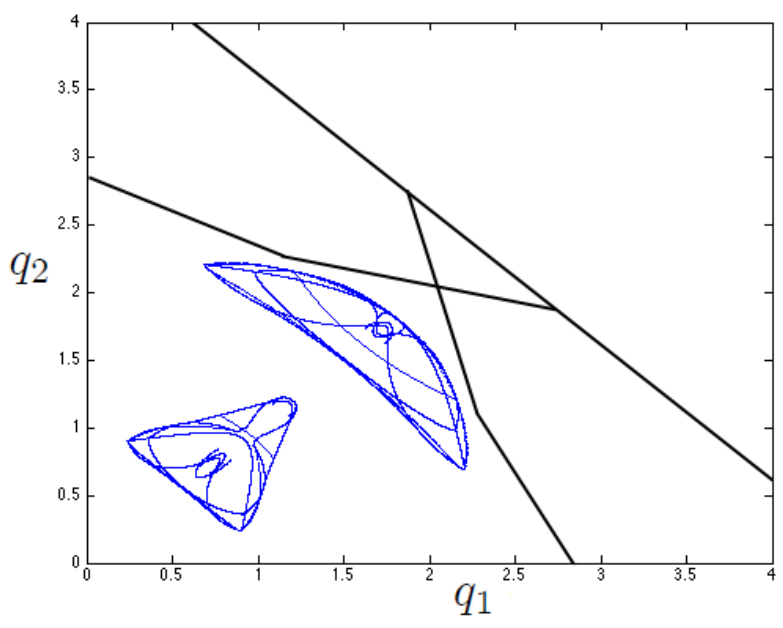

Figure 4. (a) Chaotic dynamics associated to map $T_{\Delta}$. (b) Basins of attraction of the fixed points of map $T(g=1.31)$. (c) On-off intermittency (the graph of $\left.q_{1}-q_{2}\right)$. (d) Lyapunov exponent versus parameter $g$. (e) A unique (chaotic) interior attractor captures long-term dynamics that start from blue-coloured region $(g=1.305)$. (f) Critical curves when $g=1.305$.

Case 3. Other interesting phenomena can be observed when $g \in(0,1.1]$. For small values of $g$ the absorbing area for map $T_{\Delta}$ is defined by a subset in which only the branch of map $f_{1}$ is involved. In particular, chaotic attractors or cycles of various period that capture all the dynamics that start from $\Omega_{1}$ and $\Omega_{2}$ can exist. This system definitely defines a case in which both firms invest in advertising in the long term. Specifically, for $g=0.67$ a two-period cycle is the unique attractor of the system (this is shown by $P_{1}$ and $P_{2}$ Figure 5, panel a) with respect to which firms invest in advertising. When $g=0.685$ a saddle-node bifurcation creates a four-period cycle whose basin of attraction is bounded by the stable manifolds of the four-period saddle that coexists with a cycle of period two on the diagonal (see the bifurcation diagram depicted in Figure 5, panel $\mathrm{b}$ and the phase plane plotted in Figure 5, panel c for $g=0.7$, where the four-period cycle is denoted by $R_{1}$, $R_{2}, R_{3}$ and $R_{4}$ ). We note that the basin of attraction of the cycle of period four is comprised of the immediate basin and other regions of the phase plane. This phenomenon is caused by the non invertibility of the map. For $g \cong 0.725$ the four-period attracting cycle undergoes a Neimark-Sacker bifurcation that creates a four-piece quasi-periodic attractor that initially coexists with the cycle of period two on the diagonal. For $g \cong 0.738$, a period halving bifurcation occurs after which the two-period cycle on the diagonal becomes a stable fixed point that continues to coexist with the four-piece quasi-periodic attractor (see Figure 5.d plotted for $g=0.75$, where the four-piece quasi-periodic attractor is denoted by $Y_{1}, Y_{2}, Y_{3}$ and $Y_{4}$ ). When $g \cong 0.763$, a non-local bifurcation occurs around the fixed point on $\Delta$ and an attracting cycle of period two for $T$ is born (defined by two points belonging to the two different pieces of map $T_{\Delta}$ ), that coexists with the attracting fixed point on $\Delta$. This is shown in Figure 5.e, plotted for $g=0.769$, that represents the map restricted on the diagonal where it is shown that, according to different initial conditions, trajectories are captured by two different attractors, and the phase plane reported in Figure 5.f that shows the coexistence of $E^{*}$ with a two-period cycle on the diagonal $S_{1}$ and $S_{2}$ and a four-piece quasi-periodic attractor $Y_{1}, Y_{2}, Y_{3}$ and $Y_{4}$ for $g=0.769$. When $g=0.7702$ a flip bifurcation of the cycle of period 
two (transverse with respect to the diagonal) is just occurred, so that an attracting four-period cycle outside of the diagonal $\left(W_{1}, W_{2}, W_{3}\right.$ and $\left.W_{4}\right)$ coexists with $E^{*}$ and an attracting four-piece closed invariant curve (this is shown in Figure 5, panel g).

Another interesting phenomenon is obtained when the price per unit of advertising increases at $g=0.9$. In fact, for this parameter set, the basin of attraction becomes complex and a slight change in the initial conditions determines very different long-term dynamic outcomes (as pointed out in Figure 5.h), e.g. a continuous shift from positive to zero advertising investments. This is relevant from an economic point of view, especially from policy perspectives because a slight change in initial conditions due to advertising investments may determine unexpected long term outcomes. Specifically, Figure 5.h shows that an attracting fixed point on the diagonal (that lies in a region of the phase plane where advertising investments are positive) coexists with two distinct attracting twelve-period cycles (symmetric with respect to two distinct complex basins).

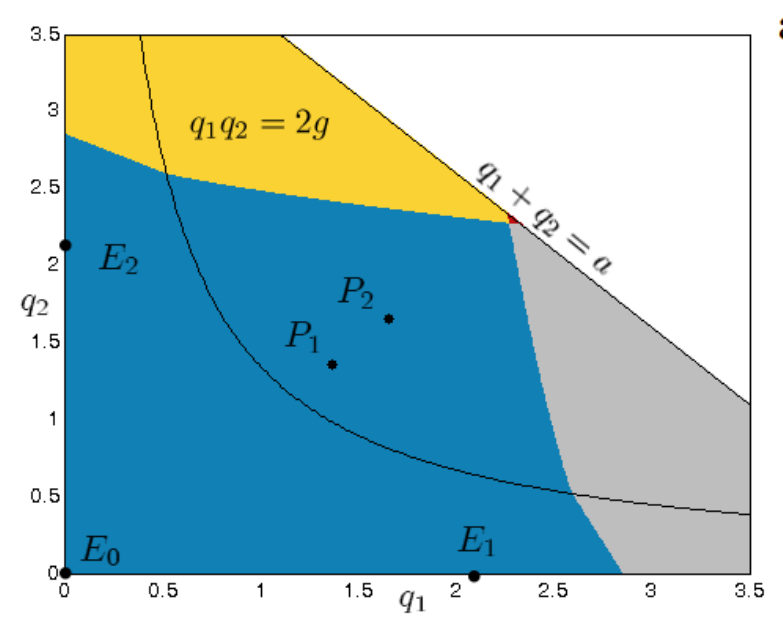

a
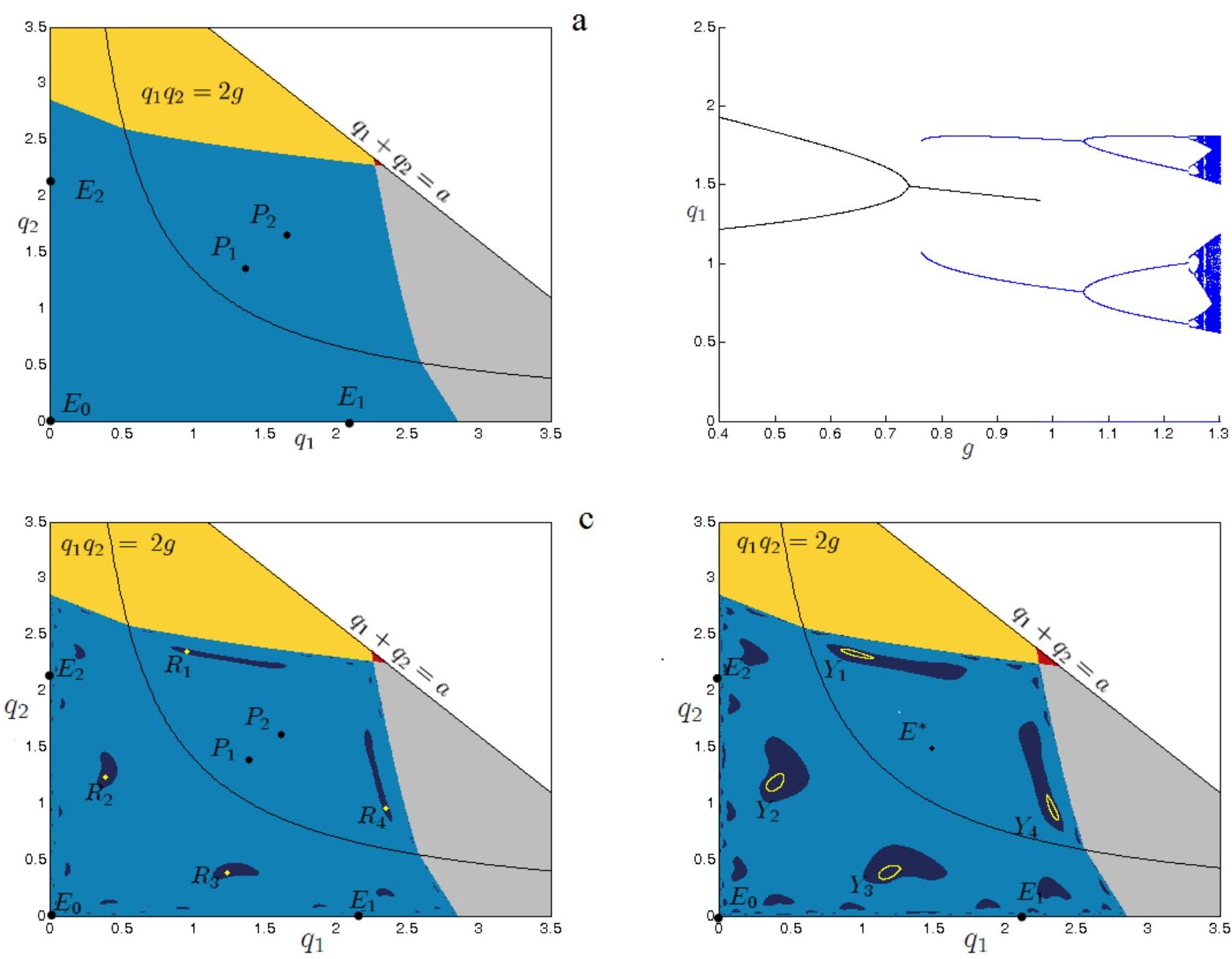

c

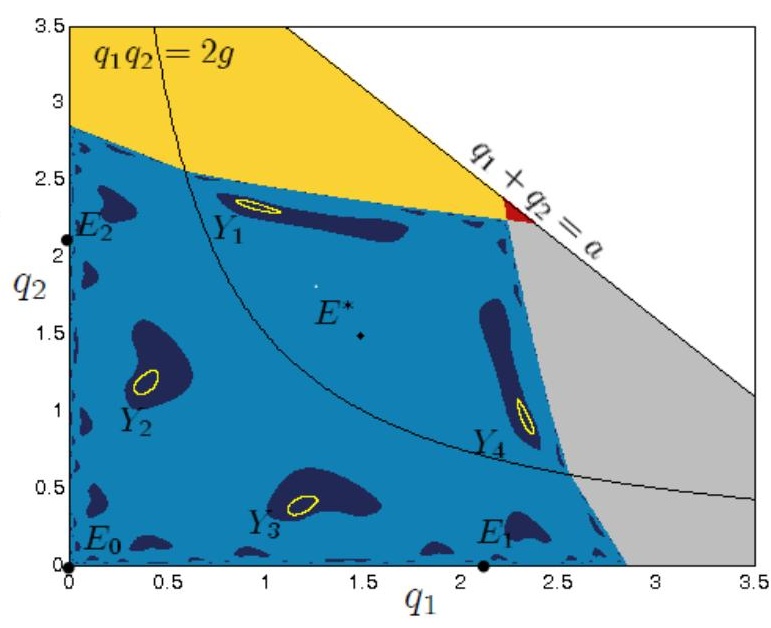



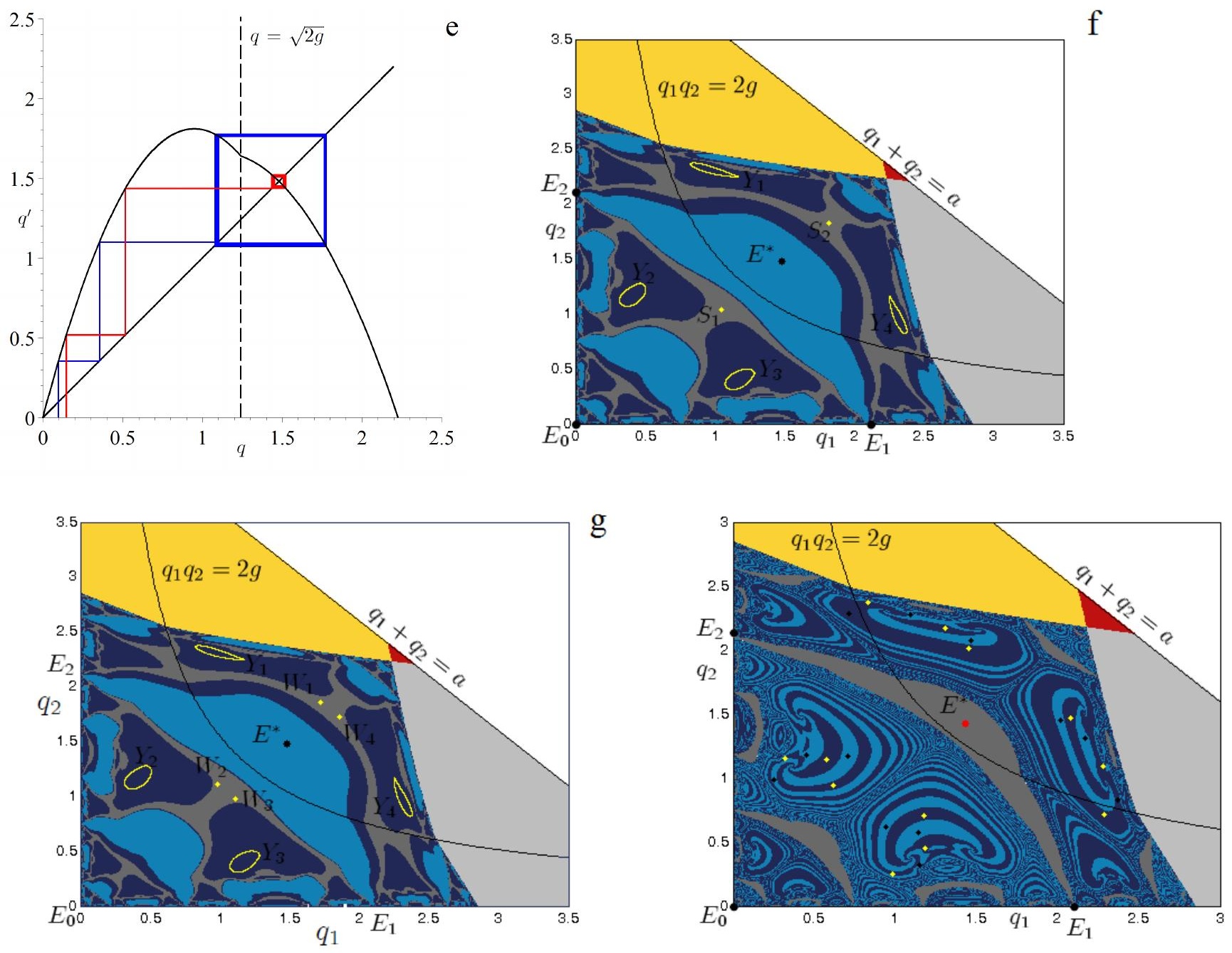

Figure 5. (a) Case $g=0.67$ (phase plane). (b) Bifurcation diagram for $g$. (c) Case $g=0.7$ (phase plane). (d) Case $g=0.75$ (phase plane). (e) Case $g=0.769$ (dynamics associated to map $T_{\Delta}$ ). (f) Case $g=0.769$ (phase plane). The fixed point $E^{*}$ coexists with an attracting two-period cycle on the diagonal and an attracting four-piece closed invariant curve born through a NeimarkSacker bifurcation. (g) Case $g=0.7702$ (phase plane). We do not show the dynamics associated to $T_{\Delta}$ in this case as the bifurcation occurs in transverse direction. (h) Case $g=0.9$ (phase plane). Tangled basins of attraction.

\section{Conclusions}

This paper has studied local and global dynamics of a nonlinear duopoly with quantity setting firms, limited information and non-cooperative (persuasive) advertising that affects the degree of 
(horizontal) products differentiation. As stressed by Bagwell (2007), advertising represents a relevant instrument for firms to make their own product different for consumers and get a competitive advantage on the market. In this context, we have found that the long-term behaviour of the economy is compatible with the existence of attractors such that firms continuously alternates between a state where there positive advertising investments to a state where there are no advertising investments, thus resembling the long-term findings of the standard Cournot duopoly (Bischi et al., 1998) in this last case. The paper has concerned with the study of both local and global behaviours of a two-dimensional dynamic discrete time system. The global study of the map has allowed us to highlight some relevant properties such as chaos synchronisation and multistability. We have shown that the choices of firms to invest or to do not invest in advertising can represent an additional cause for the non-differentiability of a map, alongside the non-differentiability induced by technology or capacity constraints already studied, for instance, by Puu and Norin (2003) and Bischi et al. (2012).

Though several analyses regarding advertising have exclusively been framed in terms of fully non-cooperative firms' interaction, a growing body of studies is focusing on semi-collusion (Fershtman and Gandal, 1994; Brod and Shivakumar, 1999; Simbanegawi, 2009). For example, when firms compete on multiple dimensions (e.g. quantity or price and advertising), semi-collusion occurs whenever economic agents choose to cooperate along some dimension(s) (advertising) while competing on another ones (price or quantity). It is important to note that in several countries semicollusion on advertising is not illegal and it may be even encouraged or mandatory. An extension of the present analysis in that direction may be part of a fruitful research agenda.

Acknowledgements The authors gratefully acknowledge Gian Italo Bischi, Riccardo Cambini, Laura Gardini and participants at AMASES 2014 held at Mediterranean University of Reggio Calabria (Italy), and MDEF 2014 held at University of Urbino (Italy). The authors also acknowledge two anonymous reviewers for comments. The usual disclaimer applies.

\section{Appendix. The static game and Nash equilibria}

Let us consider the static Cournot duopoly game developed in the main text, where firms choose advertising investments $(z)$ and quantities $(q)$ in the product market. Every player knows the market demand and is able to modify the degree of product differentiation $(d)$ through adequate investments in advertising, while also affecting the marginal willingness to pay of consumers. Therefore, player 1 solves the maximisation problem:

$$
P:\left\{\begin{array}{c}
\max _{z_{1}, q_{1}} \Pi_{1}\left(q_{1}, z_{1}, q_{2}, z_{2}\right) \\
\text { s.t. } \\
z_{1} \geq 0 \\
q_{1} \geq 0
\end{array}\right.
$$

where

$$
\Pi_{1}\left(q_{1}, z_{1}, q_{2}, z_{2}\right):=\left(a-q_{1}-\left(\frac{1}{2+2 z_{1}}+\frac{1}{2+2 z_{2}}\right) q_{2}-w\right) q_{1}-g z_{1},
$$

given the values $q_{2}$ and $z_{2}$ that player 1 expects player 2 will play. In order to avoid trivial cases, let us assume that 


\section{Assumption A.2. $a>w$.}

We note that $\Pi_{1}$ is a continuous function with respect to $q_{1}$ and $z_{1}$. However, since problem $P$ is defined on a closed and unbounded set the Weierstrass theorem on the existence of solutions of the problem cannot directly be applied. We observe, however, that since $\left(z_{1}=0, q_{1}=0\right)$ is a feasible choice, then if a maximum point does exist the value of the objective function related to it must be non negative. In addition, it is possible to show that since variables are non negative, the objective function takes negative values for $q_{1}$ or $z_{1}$ sufficiently large. In particular, since

$$
\left(a-q_{1}-\left(\frac{1}{2+2 z_{1}}+\frac{1}{2+2 z_{2}}\right) q_{2}-w\right) q_{1}-g z_{1}<\left(a-q_{1}-w\right) q_{1}-g z_{1},
$$

then for $q_{1} \geq a-w$ or $z_{1} \geq \frac{1}{g}\left(\frac{a-w}{2}\right)^{2}$ the function takes strictly negative values.

Therefore, solutions of problem $P$ coincide with solutions of the following problem:

$$
P^{\prime}:\left\{\begin{array}{c}
\max _{z_{1}, q_{1}} \Pi_{1}\left(q_{1}, z_{1}, q_{2}, z_{2}\right) \\
\text { s.t. } \\
z_{1} \geq 0 \\
q_{1} \geq 0 \\
q_{1} \leq a-w \\
z_{1} \leq \frac{1}{g}\left(\frac{a-w}{2}\right)^{2}
\end{array}\right.
$$

The optimisation problem is now defined on a rectangle, i.e. it is defined on a closed and bounded set so that a solution for $P^{\prime}$ does exist. Since $\Pi_{1}\left(q_{1}, z_{1}, q_{2}, z_{2}\right)$ takes negative values on constraints $q_{1}=a-w$ and $z_{1}=\frac{1}{g}\left(\frac{a-w}{2}\right)^{2}$ of problem (45) as well as on points $\left(z_{1}>0, q_{1}=0\right)$, its global maximum point will be located either on an interior point of the feasible region or on a point such that $\left(z_{1}=0, q_{1}>0\right)$. Since $\Pi_{1}\left(q_{1}, z_{1}, q_{2}, z_{2}\right)$ is not concave with respect to $\left(q_{1}, z_{1}\right)$, identifying the global maximum is not an easy task. Let us now proceed by using the following line of reasoning. First, we use the property (two-stage optimisation) such that given $f: U \times V \rightarrow R$ and $D \subseteq U \times V$ we have:

$$
\max _{(x, y) \in D} f(x, y)=\max _{x \in U} \max _{y \in D(x)} f(x, y),
$$

where $D(x)=\{y \in V:(x, y) \in D\}$. Since the objective function in our problem is concave with respect to $z_{1}$, it is possible to easily find the maximum point of $\Pi_{1}$ with respect to $z_{1}$. Then, the solution for $z$ is a function of $q_{1}$, expectations on $q_{2}$ as well as on parameters of the problem. Specifically, by avoiding to stress the dependency of $z_{1}$ on parameters we have that:

$$
z_{1}^{o p t}=z_{1}^{o p t}\left(q_{1}, q_{2}\right):=\left\{\begin{array}{c}
\sqrt{\frac{q_{1} q_{2}}{2 g}}-1, \text { if } q_{1} \geq \frac{2 g}{q_{2}} \\
0, \text { if } q_{1}<\frac{2 g}{q_{2}}
\end{array}=\max \left\{0, \sqrt{\frac{q_{1} q_{2}}{2 g}}-1\right\} .\right.
$$

In order to find the optimal solution we may consider the problem:

$$
\max _{q_{1} \geq 0} \widehat{\Pi}_{1}\left(q_{1}, q_{2}, z_{2}\right),
$$

where

$$
\widehat{\Pi}_{1}\left(q_{1}, q_{2}, z_{2}\right):=\left(a-q_{1}-\left(\frac{1}{2+2 z_{1}^{o p t}}+\frac{1}{2+2 z_{2}}\right) q_{2}-w\right) q_{1}-g z_{1}^{o p t}
$$


It can easily be shown that this function is of class $C^{1}$ (but in general not of class $C^{2}$ ) with respect to $q_{1}$. Therefore, if the maximum is an interior point then the first derivative evaluated at that point is null. Since the function is not concave in its domain, this condition is necessary but not sufficient. One important fact that will be useful later in the discussion is that $\widehat{\Pi}_{1}$ is concave with respect to $q_{1}$ in the interval $\left[0, \frac{2 g}{q_{2}}\right]$, that is where $z_{1}^{o p t}=0$, while when $q_{1}>2 g / q_{2}$ function $\widehat{\Pi}_{1}$ :

Case A.1 can be monotonically decreasing;

Case A.2 can admit a local maximum $\left(q_{\max }\right)$;

Case A.3 can admit a local minimum $\left(q_{\min }\right)$ and a local maximum $\left(q_{\max }\right)$ such that $q_{\min }<q_{\max }$.

When $q_{1}>2 g / q_{2}$ the sign of the first derivative can be analysed through the study of the sign of the concave function

$$
\left(\left(a-w-2 q_{1}\right)\left(1+z_{2}\right)-\frac{1}{2} q_{2}\right) \sqrt{2}-\sqrt{g} \sqrt{\frac{q_{2}}{q_{1}}}\left(1+z_{2}\right) .
$$

As a consequence, the first derivative admits at most two changes in sign (negative, positive, negative) for $q_{1}>2 g / q_{2}$. We note that there may exist expectations on the choices of the rival such that the optimal choice for firm 1 is $q_{1}=0$, so that $z_{1}=0$. In order to rule out this case we introduce the following assumption:

\section{Assumption A.3.}

$$
\left.\frac{\partial \widehat{\Pi}_{1}\left(q_{1}, q_{2}, z_{2}\right)}{\partial q_{1}}\right|_{q_{1}=0}=a-\left(\frac{1}{2}+\frac{1}{2+2 z_{2}}\right) q_{2}-w>0 .
$$

From (51) we have that at the optimum $q_{1}>0$ and profits are positive while advertising investments can be positive or null. The following claims summarise our findings.

Claim 8 If $\left.\frac{\partial \widehat{\Pi}_{1}\left(q_{1}, q_{2}, z_{2}\right)}{\partial q_{1}}\right|_{q_{1}=\frac{2 g}{q_{2}}}>0$, given the concavity of $\widehat{\Pi}_{1}$ in the interval $\left[0, \frac{2 g}{q_{2}}\right], \widehat{\Pi}_{1}$ results to be monotonically increasing and in $\left[0, \frac{2 g}{q_{2}}\right]$ and then a local optimum does not exist in such an interval. Therefore, the optimum for firm 1 will involve a strictly positive value of $z_{1}$. The optimum can easily be found since when $q_{1}>2 g / q_{2}$ only one critical point does exist (Case A.2 above).

Claim 9 If $\left.\frac{\partial \widehat{\Pi}_{1}\left(q_{1}, q_{2}, z_{2}\right)}{\partial q_{1}}\right|_{q_{1}=\frac{2 g}{q_{2}}}<0$, a local optimum for the objective function does exist in the interval $\left[0, \frac{2 g}{q_{2}}\right]$. However, this does not guarantee that it is the global optimum (i.e., the solution of the optimisation problem). It is also the global optimum if Case A.1 above holds. In contrast, if Case A.3 holds the global optimum can be identified only numerically at each of the two local maxima. 
Equilibrium. From the analysis above, it is clear that if Nash equilibria exist will be such that marginal profits of both firms are simultaneously zero, that is:

$$
\left\{\begin{aligned}
\frac{\partial \Pi_{1}\left(q_{1}, z_{1}, q_{2}, z_{2}\right)}{\partial q_{1}}=0 \\
\frac{\partial \Pi_{1}\left(q_{1}, z_{1}, q_{2}, z_{2}\right)}{\partial z_{1}}=0 \\
\frac{\partial \Pi_{2}\left(q_{2}, z_{2}, q_{1}, z_{1}\right)}{\partial q_{2}}=0 \\
\frac{\partial \Pi_{2}\left(q_{2}, z_{2}, q_{1}, z_{1}\right)}{\partial z_{2}}=0
\end{aligned}\right.
$$

or equivalently

$$
\left\{\begin{array}{c}
\frac{\partial \widehat{\Pi}_{1}\left(q_{1}, q_{2}, z_{2}\right)}{\partial q_{1}}=0 \\
\frac{\partial \widehat{\Pi}_{2}\left(q_{2}, q_{1}, z_{1}\right)}{\partial q_{2}}=0 \\
z_{1}=z_{1}^{o p t}\left(q_{1}\right) \\
z_{2}=z_{2}^{o p t}\left(q_{2}\right)
\end{array}\right.
$$

Since the optimal rule for $z$ is the same for both firms, a Nash equilibrium will imply that either firms will invest the same amount of resources in advertising (positive or zero). Then, we can split (52) in the following two systems:

$$
\left\{\begin{array}{l}
a-w-2 q_{1}-\sqrt{\frac{2 g q_{2}}{q_{1}}}=0 \\
a-w-2 q_{2}-\sqrt{\frac{2 g q_{1}}{q^{2}}}=0 \quad, \quad \text { if } \quad q_{1} q_{2} \geq 2 g \\
z_{1}^{o p t}=z_{2}^{o p t}=\sqrt{\frac{q_{1} q_{2}}{2 g}}-1
\end{array}\right.
$$

and

$$
\left\{\begin{array}{c}
a-w-2 q_{1}-q_{2}=0 \\
a-w-2 q_{2}-q_{1}=0 \quad, \quad \text { if } \quad q_{1} q_{2}<2 g . \\
z_{1}^{o p t}=z_{2}^{o p t}=0
\end{array}\right.
$$

System in (54) has the unique solution

$$
N^{A D V}:=\left(\frac{a-w-\sqrt{2 g}}{2}, \frac{a-w}{4} \sqrt{\frac{2}{g}}-\frac{3}{2}, \frac{a-w-\sqrt{2 g}}{2}, \frac{a-w}{4} \sqrt{\frac{2}{g}}-\frac{3}{2}\right),
$$

which is feasible if and only if $g \leq \frac{(a-w)^{2}}{18}$. In contrast, system in (55) has the unique solution:

$$
N^{N O A D V}:=\left(\frac{a-w}{3}, 0, \frac{a-w}{3}, 0\right)
$$

which is feasible if and only if $g>\frac{(a-w)^{2}}{18}$.

Theorem 10 If $g \leq \frac{(a-w)^{2}}{18}$ then $N^{A D V}$ is the Nash equilibrium of the game. If $g>\frac{(a-w)^{2}}{18}$ then $N^{N O A D V}$ is the Nash equilibrium of the game. 
Proof. By the study of the Hessian matrix of $\Pi_{1}\left(q_{1}, z_{1}, q^{*}, z^{*}\right)$ we have that $\left(q_{1}, z_{1}\right)=\left(q^{*}, z^{*}\right)$ is still a local maximum, that is firm 1 cannot be better off (improve profits) by shifting $q_{1}$ and $z_{1}$ quite slightly, where

$$
q^{*}=\left\{\begin{array}{c}
\frac{a-w-\sqrt{2 g}}{2}, \quad \text { if } g \leq \frac{(a-w)^{2}}{18} \\
\frac{a-w}{3}, \quad \text { if } g>\frac{(a-w)^{2}}{18}
\end{array},\right.
$$

and

$$
z^{*}=\left\{\begin{array}{c}
\frac{a-w}{4} \sqrt{\frac{2}{g}}-\frac{3}{2}, \quad \text { if } g \leq \frac{(a-w)^{2}}{18} \\
0, \quad \text { if } g>\frac{(a-w)^{2}}{18}
\end{array} .\right.
$$

In what follows we show that the local maximum is also a global maximum, that is there exist no alternatives for firms with higher profits than those identified in (56) for $g \leq \frac{(a-w)^{2}}{18}$ and (57) for $g>\frac{(a-w)^{2}}{18}$. Let us now consider

$$
\widehat{\Pi}_{1}\left(q_{1}, q^{*}, z^{*}\right),
$$

and verify that $q=q^{*}$ results to be the global optimum. The problem is solved by considering two distinct cases.

a) Case $g \leq \frac{(a-w)^{2}}{18}$ (positive advertising investment). If $q_{1}=q^{*}$ (and then $z_{1}=z^{*}=z_{1}^{o p t}\left(q^{*}, q^{*}\right)$ ) is not optimal for player 1 then there should be another maximum $q_{1}=q^{* *}$ for $(60)$, that is for the function:

$$
\widehat{\Pi}_{1}\left(q_{1}, q^{*}, z^{*}\right)=\left(a-q_{1}-\left(\frac{1}{2\left(1+z_{1}^{o p t}\left(q_{1}, q^{*}\right)\right)}+\frac{1}{2\left(1+z^{*}\right)}\right) q_{2}^{*}-w\right) q_{1}-g z_{1}^{o p t}\left(q_{1}, q^{*}\right) .
$$

We note that (61) is of class $C^{1}$ with $z_{1}^{\text {opt }}\left(q_{1}, q^{*}\right)=0$ for $q_{1}<2 g / q^{*}$. From Claim 8, Claim 9 and the fact that $q^{*}$ is a local maximum for $\widehat{\Pi}_{1}$ then $q^{* *}$ cannot belong to $\left[2 g / q^{*}, a-w\right]$. In addition, since $\lim _{q_{1} \rightarrow\left(2 g / q^{*}\right)^{-}} \frac{\partial \widehat{\Pi}_{1}\left(q_{1}, q^{*}, z^{*}\right)}{\partial q_{1}}>0$ we have also that $q^{* *}$ cannot belong to $\left[0,2 g / q^{*}\right)$. Therefore, $q^{*}$ is the optimal choice for firm 1 and given the symmetry of players quadruple $\left(q^{*}, z^{*}, q^{*}, z^{*}\right)$ is a Nash equilibrium.

b) Case $g>\frac{(a-w)^{2}}{18}$ (no advertising investment). Since a maximum point does exist in the interval $\left[0,2 g / q^{*}\right)$,

$$
\lim _{q_{1} \rightarrow\left(2 g / q^{*}\right)^{+}} \frac{\partial \widehat{\Pi}_{1}\left(q_{1}, q^{*}, z^{*}\right)}{\partial q_{1}}<0,
$$

and

$$
\operatorname{sgn}\left\{\frac{\partial \widehat{\Pi}_{1}\left(q_{1}, q^{*}, z^{*}\right)}{\partial q_{1}}\right\}=\operatorname{sgn}\left\{5(a-w) \sqrt{q_{1}}-12 q_{1} \sqrt{q_{1}}-\sqrt{6 g(a-w)}\right\},
$$

it can easily be concluded that $\frac{\partial \widehat{\Pi}_{1}\left(q_{1}, q^{*}, z^{*}\right)}{\partial q_{1}}<0$ for any $q_{1} \in\left[2 g / q^{*}, a-w\right]$. Then, given the symmetry of players it follows that quadruple $\left(q^{*}, 0, q^{*}, 0\right)$ is a Nash equilibrium. 


\section{References}

Ahmed, E., Agiza, H.N., Hassan, S.Z., 1999. On modelling advertisement in Cournot duopoly. Chaos, Solitons \& Fractals 10, 1179-1184.

Bagwell, K., 2007. The economic analysis of advertising. Handbook of Industrial Organization 3, 1701-1844.

Bischi, G.I., Kopel, M., 2003. Multistability and path dependence in a dynamic brand competition model. Chaos, Solitons \& Fractals 18, 561-576.

Bischi, G.I., Naimzada, A., 2000. Global analysis of a dynamic duopoly game with bounded rationality. In: Filar, J.A., Gaitsgory, V., Mizukami, K., Eds. Advances in Dynamics Games and Application 5. Boston: Birkhäuser, 361-385.

Bischi, G.I., Gardini, L., Kopel, M., 2000. Analysis of global bifurcations in a market share attraction model. Journal of Economic Dynamics \& Control 24, 855-879.

Bischi, G.I., Lamantia, F., Sushko, I., 2012. Border collision bifurcations in a simple oligopoly model with constraints. International Journal of Applied Mathematics \& Statistics 26, 121-135.

Bischi, G.I., Stefanini, L., Gardini, L., 1998. Synchronization, intermittency and critical curves in a duopoly game. Mathematics and Computers in Simulation 44, 559-585.

Bischi, G.I., Chiarella. C., Kopel. M., Szidarovszky. F., 2010. Nonlinear Oligopolies. Stability and Bifurcations. Berlin, Heidelberg: Springer-Verlag.

Brod, A., Shivakumar, R., 1999. Advantageous semicollusion. Journal of Industrial Economics 47, 221-230.

Colangelo, G., 1992. Controllo verticale, pubblicità e incentivi alla fusione orizzontale. Economia Politica 9, 405-423.

Dixit A.K, Norman, V., 1978. Advertising and welfare. Bell Journal of Economics 9, 1-17.

Fanti, L., Gori, L., Sodini, M., 2012. Nonlinear dynamics in a Cournot duopoly with relative profit delegation. Chaos, Solitons \& Fractals 45, 1469-1478.

Fanti, L., Gori, L., Sodini, M., 2013. Nonlinear dynamics in a Cournot duopoly with different attitudes towards strategic uncertainty. Abstract and Applied Analysis 2013, Article ID 323290.

Fershtman, C. Gandal, N., 1994. Disadvantageous semicollusion. International Journal of Industrial Organization 12, 141-154.

Grossman, G., Shapiro, C., 1984. Informative advertising with differentiated products. Review of Economic Studies 51, 63-81.

Häckner, J., 2000. A note on price and quantity competition in differentiated oligopolies. Journal of Economic Theory 93, 233-239.

Hamilton, S.F., 2009. Informative advertising in differentiated oligopoly markets. International Journal of Industrial Organization 27, 60-69.

Kopel, M., Lambertini, L., 2013. On price competition with market share delegation contracts. Managerial and Decision Economics 34, 40-43.

Luhta, I., Virtanen, I., 1996. Non-linear advertising capital model with time delayed feedback between advertising and stock of goodwill. Chaos, Solitons \& Fractals 7, 2083-2104.

Puu, T., Norin, A., 2003. Cournot duopoly when the competitors operate under capacity constraints. Chaos, Solitons \& Fractals 18, 577-592.

Radi, D., Gardini, L., Avrutin, V., 2014. The role of constraints in a segregation model: the symmetric case. Chaos, Solitons \& Fractals 66, 103-119.

Simbanegawi, W., 2009. Informative advertising: competition or cooperation? Journal of Industrial Economics 57, 147-166. 
Singh, N., Vives, X., 1984. Price and quantity competition in a differentiated duopoly. RAND Journal of Economics 15, 546-554.

Stahl, D.O., 1994. Oligopolistic pricing and advertising. Journal of Economic Theory 64, 162177 . 